\title{
Highly Dispersive Nearly Free Electron Bands at a 2D-assembled C60 Monolayer
}

\author{
Masahiro Shibuta, ${ }^{\dagger, \star \S}$ Kazuo Yamamoto, ${ }^{\ddagger}$ Hongli Guo, ${ }^{\|}$Jin Zhao, and Atsushi Nakajima ${ }^{\dagger, \star \star *}$
}

${ }^{\dagger}$ Keio Institute of Pure and Applied Science (KiPAS), and ${ }^{\star}$ Department of Chemistry, Faculty of Science and Technology, Keio University, 3-14-1 Hiyoshi, Kohoku-ku, Yokohama 223-8522, Japan

${ }^{\S}$ Fachbereich Physik und Zentrum für Materialwissenschaften, Philipps-Universität, D-35032 Marburg, Germany

"ICQD/Hefei National Laboratory for Physical Sciences at Microscale and Key Laboratory of StronglyCoupled Quantum Matter Physics, Chinese Academy of Sciences, and Department of Physics, University of Science and Technology of China, Hefei, Anhui 230026, China

*Addresses correspondence to

Tel: +81-45-566-1712, Fax: +81-45-566-1697

E-mail: nakajima@chem.keio.ac.jp

Table of contents

Note S1. Coverage determination.

(Page S2)

Figure S1. Coverage dependence of 2PPE spectra.

Note $\mathrm{S2}$. Electron excitation mechanism at $\mathrm{C}_{60} / \mathrm{HOPG}$ system.

Figure S2. 2PPE spectrum for $\mathrm{C}_{60}$ monolayer on $\mathrm{Au}(111)$ substrate.

Figure S3. Calculated full band map for 2D-assembled $\mathrm{C}_{60}$ monolayer.

Figure S4. Calculated orbital distribution of bands 148 and 151. 


\section{Note S1. Coverage determination of a $\mathbf{C}_{60}$ film}

To obtain clear and reliable dispersions of SAs as shown in the main text, preparation of a wellordered $\mathrm{C}_{60}$ monolayer (ML) film is required. Therefore, we carefully deposited $\mathrm{C}_{60}$ molecules with as slow a growth rate as possible and calibrated the deposition amount of $\mathrm{C}_{60}$. Figure $\mathrm{S} 1$ shows the coverage dependence of 2PPE spectra ( $h v=4.33 \mathrm{eV}$, normal emission) for the $\mathrm{C}_{60}$ deposited on HOPG, where the horizontal axis stands for the excited state energy by absorption of $h v$ with respect to the Fermi level $\left(E_{\mathrm{F}}\right)$. For the clean $\mathrm{HOPG},{ }^{1,2}$ a broad structure due to a $\pi^{*}$ band of graphite is observed at $E_{\mathrm{F}}+1.6 \mathrm{eV}$. At $E_{\mathrm{F}}+$ $3.58 \mathrm{eV}$, a sharp peak is also recognized originating from the first image potential state (IPS) formed on a clean HOPG labeled as IPSHOPG. From the low-energy cutoff of the spectrum, the vacuum level, $E_{\text {vac, }}$ for the clean HOPG is determined to be $E_{\mathrm{F}}+4.47 \mathrm{eV}$ ( $h v+$ cut off energy).

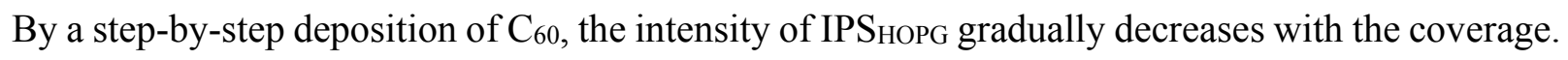
From the attenuation of IPSHOPG, we defined the deposition of $\mathrm{C}_{60}$ for $14 \mathrm{~min}$ corresponding to $1.0 \mathrm{ML}$ as denoted on the right-hand side. Consistently, the $E_{\text {vac }}$ shifted toward a higher energy and mostly saturated at $1 \mathrm{ML}\left(E_{\mathrm{vac}}=4.60 \mathrm{eV}\right)$.
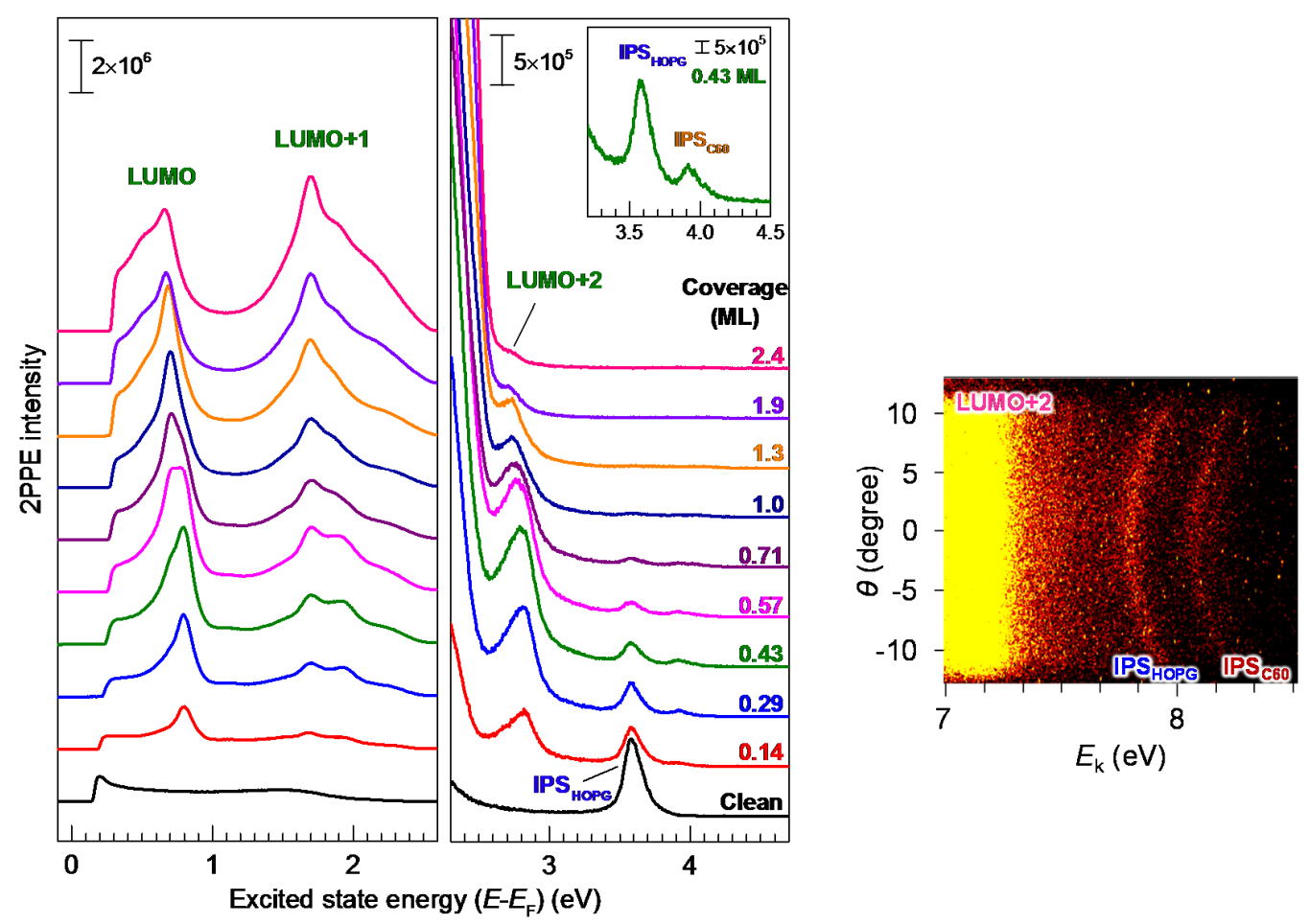

Figure S1. Coverage dependence of the 2PPE spectra at normal emission (left). Angular distribution of 2PPE for a $0.43 \mathrm{ML} \mathrm{C}_{60}$ film on HOPG (right). The photon energy is $4.33 \mathrm{eV}$. 


\section{Note S2. Electron excitation mechanism for the $\mathrm{C}_{60} / \mathrm{HOPG}$ system}

On the right-hand side of Figure $\mathrm{S} 1$, by the deposition of $\mathrm{C}_{60}$ molecules, the LUMO and LUMO+1 derived features appear at a lower energy region, as discussed in the main text and previous reports. ${ }^{2,3} \mathrm{~A}$ fine structure of LUMO+1 originates from the resonance excitations of SAs (see main text). At a higher energy region (Figure $\mathrm{S} 1$, right), a peak at $E_{\mathrm{F}}+2.8 \mathrm{eV}$ appears by increasing the $\mathrm{C}_{60}$ coverage, which originates from LUMO+2 where the energy level locates at $2.1 \mathrm{eV}$ above LUMO from the inverse photoemission spectroscopy. ${ }^{4}$ The intensity of LUMO+2 is much weaker than those for LUMO or LUMO+1 (involving SAs) by a factor of $\sim 1 / 5$. Furthermore, the intensity of LUMO +2 begins to decrease at about one-half ML. These behaviors can be explained by the electron excitation process in the $\mathrm{C}_{60} / \mathrm{HOPG}$ system. The $\mathrm{LUMO}+2$ appears for the $\mathrm{C}_{60}$ film on an $\mathrm{Au}(111)$ substrate with comparable intensity together with LUMO and LUMO+1 (see Figure S2). The above discussion on the electron excitation processes depending on the substrate was reported in Refs. 2 and 3.

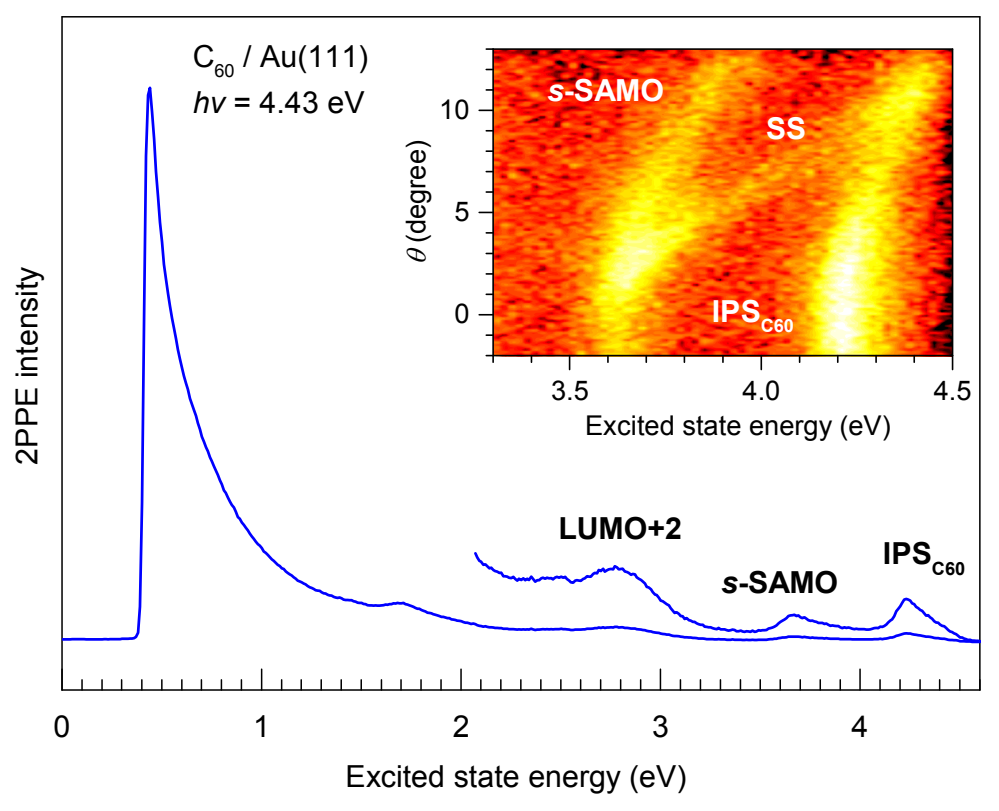

Figure S2. Angular integrated 2PPE spectra $(h v=4.43 \mathrm{eV})$ for a $\mathrm{C}_{60}$ monolayer film on $\mathrm{Au}(111)$. A peak at $E_{\mathrm{F}}+2.8 \mathrm{eV}$ originates from LUMO+2, which is very weakly resolved in a HOPG substrate at the same energy (Figure S1). The spectral features at higher energy $\left(E_{\mathrm{F}}+3 \sim 4.5 \mathrm{eV}\right)$ show clear angular distributions as shown in the inset where three NFE bands exist in the energy window. According to previous reports, the NFE bands are attributable to $s$-SAMO, Shockley surface state (SS), ${ }^{5}$ and IPS on $\mathrm{C}_{60}$ (IPS 60 ), as denoted in the figure. The energy of $s$-SAMO at $\theta=0^{\circ}$ ( $\Gamma$ point) is $E_{\mathrm{F}}+3.63 \mathrm{eV}$, which is nearly identical with the reported value $\left(E_{\mathrm{F}}+3.65 \mathrm{eV}\right)$. The 2PPE signal at a lower energy region is governed by secondary electrons or hot electrons, which make the spectral feature of SAMOs more vague. 
On the other hand, the peak at $E_{\mathrm{F}}+3.90 \mathrm{eV}$ is assignable to the IPS formed on a $\mathrm{C}_{60}$ substrate denoted as IPS 60 in which the assignment has been confirmed by the observations featuring the IPS, including an NFE dispersion obtained by AR-2PPE (Figure S1). The IPS 60 signals mostly disappear at a higher coverage greater than $1 \mathrm{ML}$, revealing the photoexcitation mechanism of the $\mathrm{C}_{60} / \mathrm{HOPG}$ interface. Specifically, the photoexcited electrons just below the $E_{\mathrm{F}}$ can transfer through a $\mathrm{C}_{60} / \mathrm{Au}$ interface but less transfer through a $\mathrm{C}_{60} / \mathrm{HOPG}$ interface. $^{3}$

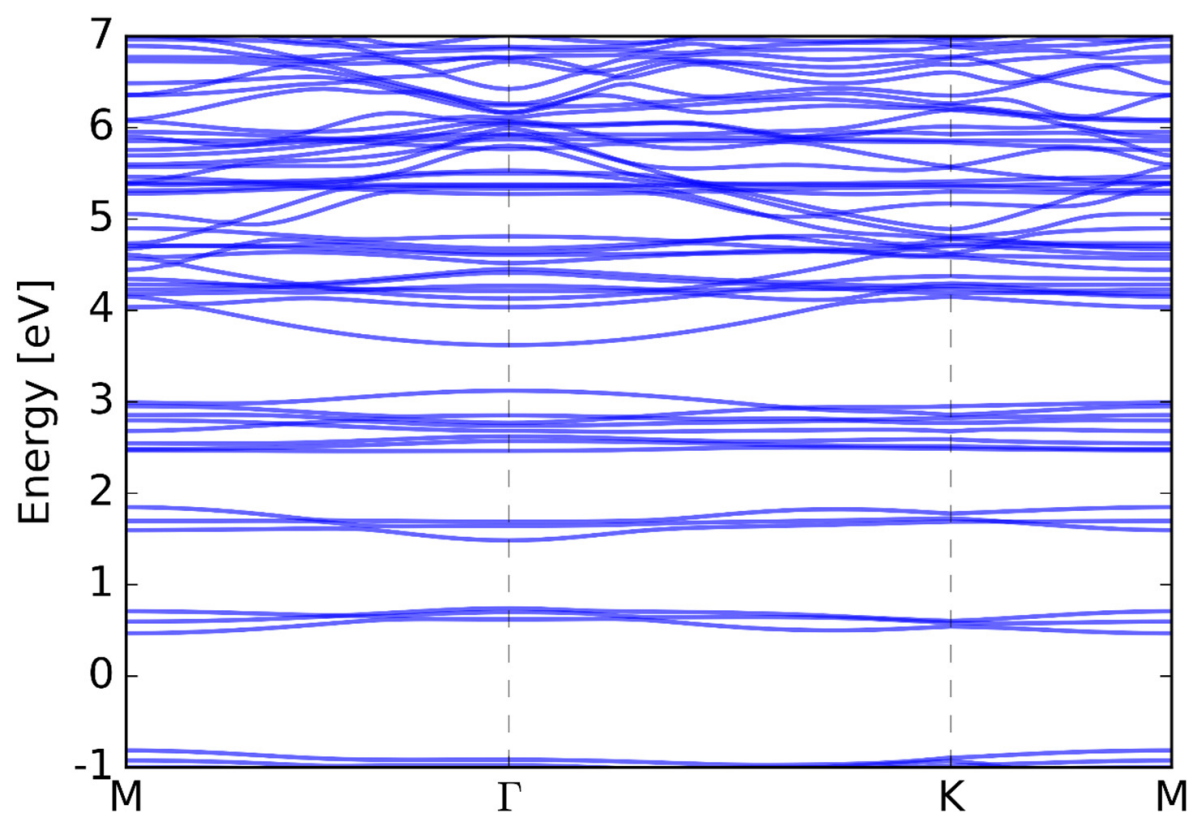

Figure S3. The calculated full band map for a 2D-assembled $\mathrm{C}_{60}$ monolayer above the HOMO-derived bands. 
(a) Band 148

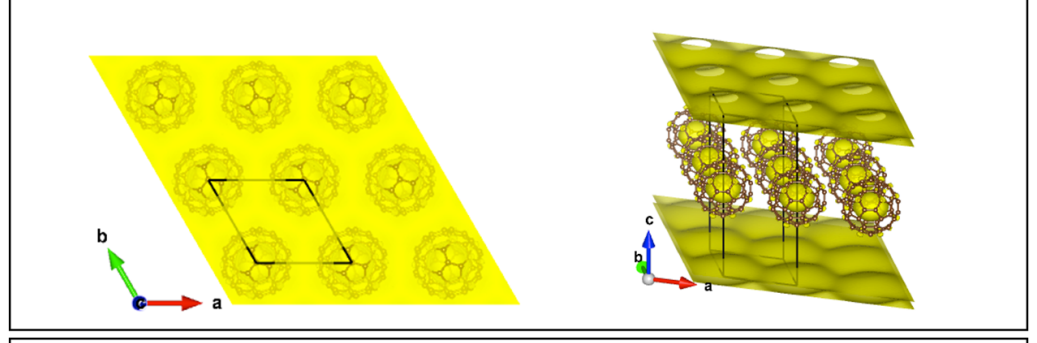

(b) Band 151
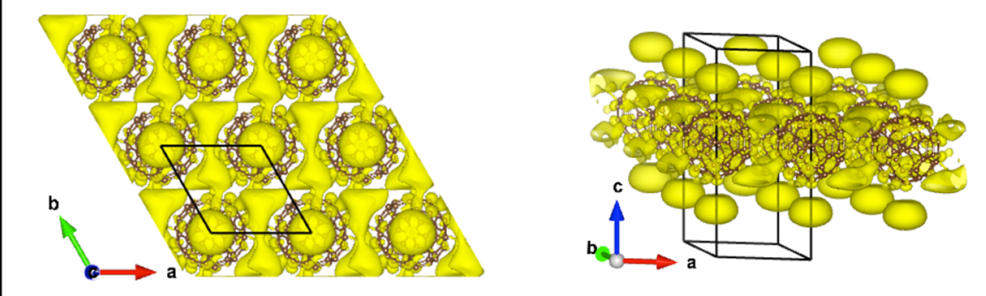

Figure S4. The calculated orbital distribution of a 2D-assembled $\mathrm{C}_{60}$ monolayer for (a) band 148 and (b) band 151. Both bands contribute to less-dispersive LA and LB, respectively, obtained by AR-2PPE (Figure 2b). Although these calculated bands also exhibit small dispersions around the gamma point, as shown in Figure 4, the orbital distributions reveal their diffuse characters with SAMOs.

\section{References}

(1) Shibuta, M.; Yamamoto, K.; Miyakubo, K.; Yamada, T.; Munakata, T. Resonant Effects on TwoPhoton Photoemission Spectroscopy: Linewidths and Intensities of Occupied and Unoccupied Features for Lead Phthalocyanine Films on Graphite. Phys. Rev. B 2010, 81, 115426.

(2) Shibuta M.; Yamamoto, K.; Yamagiwa, K.; Eguchi, T.; Nakajima, A. Photoexcited Electron Transfer Properties at $\mathrm{C}_{60}$ Film on Graphite and on $\mathrm{Au}(111)$ Interfaces Studied by Two-Photon Photoemission Spectroscopy. Chem. Lett. 2017, 46, 1528-1531.

(3) Shibuta, M.; Yamamoto, K.; Ohta, T.; Nakaya, M.; Eguchi, T.; Nakajima, A. Direct Observation of Photocarrier Electron Dynamics in $\mathrm{C}_{60}$ Films on Graphite by Time-Resolved Two-Photon Photoemission. Sci. Rep. 2016, 6, 35853.

(4) Lof, R. W.; Van Veenendaal, M. A.; Koopmans, B.; Jonkman, H. T.; Sawatzky, G. A. Band Gap, Excitons, and Coulomb Interaction in Solid C60. Phys. Rev. Lett. 1992, 68, 3924-3927.

(5) Chan, W.-L.; Tritsch, J.; Dolocan, A.; Ligges, M.; Miaja-Avila, L.; Zhu X.-Y. Momentum-Resolved Quantum Interference in Optically Excited Surface States. J. Chem. Phys. 2011, 135, 031101. 